\title{
Evidence against independent processing of black and white pattern features
}

\author{
PATRICK CAVANAGH \\ Université de Montréal, Montreal, Quebec H3C 3J7, Canada \\ and \\ EDWARD M. BRUSSELL and STEPHEN R. STOBER \\ Concordia University, Montreal, Quebec H3G IM8, Canada
}

\begin{abstract}
Following adaptation to a rectangular grating, subjects observed a shift in the perceived duty cycle of a test grating (Burton, Nagshineh, \& Ruddock, 1977; DeValois, 1977). This suggests that size information about black and white pattern components may be analyzed independently. We have tested this hypothesis by combining adaptation to a 2-cpd rectangular wave grating (narrow white bars and wide black bars or vice versa) with adaptation to a 4-cpd sine wave (narrow white and black bars). The data do not support the independent analysis of white and black components, but they may be accounted for by mechanisms sensitive to the relative phase relations among the spatial frequency components of the input.
\end{abstract}

Recently, DeValois (1977) has demonstrated that adaptation to a rectangular wave grating produces a shift in the perceived duty cycle of a square wave test grating. A few seconds' inspection of the left-hand pair of gratings in Figure 1, fixating on the central dots, will produce the effect when the right-hand gratings are viewed.

DeValois' data led her to suggest that black and white size-specific information may be processed separately in the visual system or, alternatively, in Fourier terms, spatial frequency channels must be phase specific. In the first case, there would be two separate, contrast-specific populations of size-tuned bar detectors: one for detecting white bars and the other for detecting black bars. Prolonged observation of the upper left-hand grating of Figure 1 would therefore adapt detectors tuned to narrow white bars as well as detectors tuned to wide black bars. Since this adaptation will skew the distribution of detectors responding to subsequently viewed bars, the central tendency of the distribution responding to the white bars in the test grating will shift toward wide size-tuned units, while the distribution for the black bars will shift toward narrower units. Burton, Nagshineh, and Ruddock (1977) have replicated DeValois' (1977) results and have extended the model of independent black and white detectors to cover all possible combinations of adapt and test bar widths, including those of the frequency shift paradigm (Blakemore \& Sutton, 1969). In particular, Burton et al. (1977) show that the perceived width of either the black or white bars in a test

This research was supported by Grants A8606 and A9937 from the Natural Sciences and Engineering Research Council of Canada and from the Ministère de l'Education du Québec.
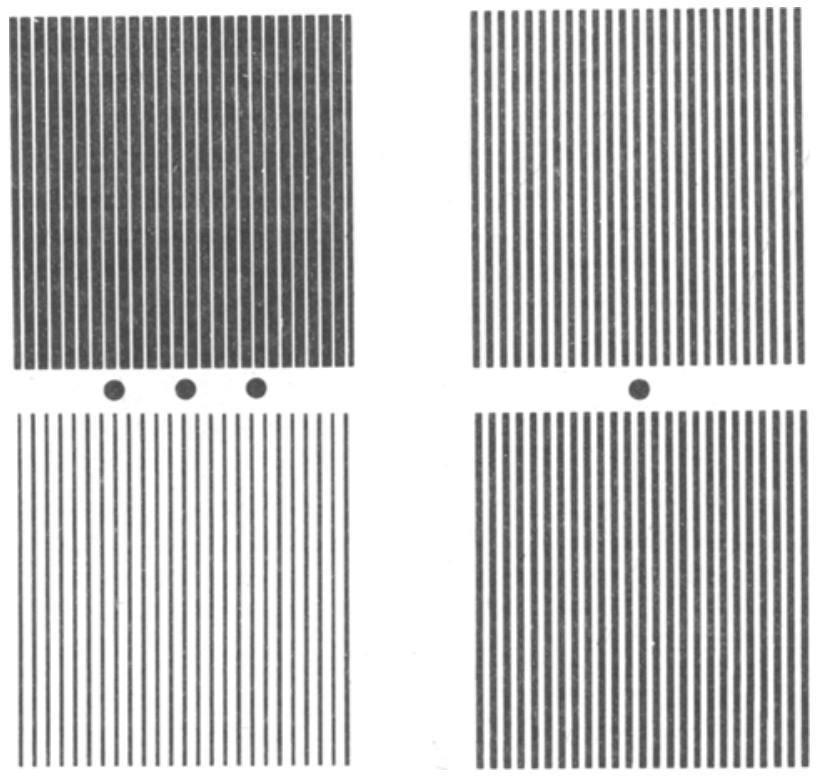

Figure 1. Demonstration of the duty cycle shift produced by either of the rectangular gratings. See text for instructions.

grating can be changed independently of the width of the bars of opposite contrast in the test or adaptation gratings. For example, varying the width of the white bars in the adapting grating appears to affect the perceived width of only the white bars in the test grating, and the effect is invariant to the widths of the black bars in either the test or adaptation gratings. Burton et al. conclude that the simplest interpretation of their data is that black and white information is processed independently.

The simplicity of the interpretation is no guarantee 
of its correctness, however, and alternative explanations based on the encoding of relative phase (Arend \& Lange, 1979; Atkinson \& Campbell, 1974; Burr, 1980; DeValeis, 1977; Nachmias \& Weber, 1975; Sansbury, Distelhorst, \& Moore, 1978; Stromeyer, Lange, \& Ganz, 1973) between the harmonic components of the gratings may be possible. The presence of a wide white grating, for example, would be signaled by a phase-sensitive channel responding maximally to the grating's first and second harmonics in peaks-subtract phase; a wide black grating would be signaled by a channel responding maximally to the first and second harmonics in peaks-add phase. Following adaptation to a rectangular grating of a given duty cycle, there would be an imbalance in the distribution of responsiveness across the phase-sensitive channels that respond to first and second harmonics. A test square wave grating (which requires a balance across these particular channels in order to be perceived as square) would then appear to have a duty cycle shifted away from $50 \%$ in the direction opposite to that of the adapting grating.

We have tested these two models by adapting to rectangular wave gratings alternating with sine wave gratings of twice the frequency. In order to evaluate the separate predictions that the models make for black and white bars under these conditions, we used a procedure that allows us to measure bar-width shifts for black and white bars independently. Figure 2 shows the two adaptation fields for a $25 \%$ duty cycle rectangular grating.

The two adaptation fields in Figure 2 both present narrow white bars, but the black bars are narrow in the Field 2 (2f) grating and wide in the rectangular grating. We would expect, according to the independent processing model, that the effects of the adaptations would counteract each other for the black bars

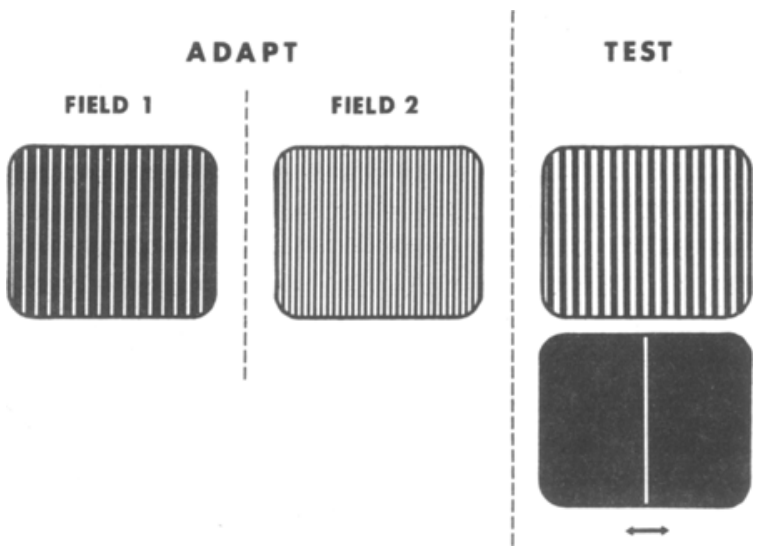

Figure 2. Bar adjustment procedure following combined adaptation. The two adapting fields were presented in an alternating fashion at the same spatial location. The test grating was also presented at the same location, with the adjustable bar immediately below. Adapting Field 2 and the test field were actually sine wave gratings. but support each other for the white bars. (Note that both adapting Field 2 and the test field were actually sine wave gratings and are presented here as square waves for convenience. Sine wave test gratings were used to avoid any possible interactions between the upper harmonics of the rectangular grating and those of a square wave test.)

In the test phase, a single bar is placed below a bar of the same contrast in the inspection grating, and the subject adjusts the width of the single bar to match the perceived width of the inspection bar above it. In Figure 2, then, we expect a significant barwidth shift for the white bar but little or none for the black bar. Conversely, for a $75 \%$ rectangular wave grating, a shift would be expected for the black bar but not for the white.

The prediction based on the hypothesis of phasesensitive channels is centered on the fact that the second adapting field, alternating with the rectangular grating, is at the frequency of the second harmonic of the rectangular grating. In this model, duty cycle shift is a result of the imbalance in the distribution of channels tuned to the first and second harmonics at various relative phases. If there is some linearity in the phase-sensitive mechanism, then all channels responding to the first and second harmonics in various phase combinations must also respond to the second harmonic in isolation. Adaptation to the second harmonic should therefore reduce the responsiveness of all these phase-sensitive channels uniformly, reducing or eliminating the imbalance caused by the adaptation to the rectangular grating. The hypothesis of phase-sensitive mechanisms therefore predicts that the differential shifts in black and white bar widths caused by adaptation to a rectangular wave grating will be suppressed by additional adaptation to a $2 \mathrm{f}$ grating.

Before running this combined adaptation experiment, two control experiments were run to evaluate our procedure of using sine rather than square wave and intermittent rather than steady adaptation and to determine the effects of the various adaptation stimuli when presented alone.

\section{METHOD}

\section{Equipment and Stimuli}

In an otherwise dark room, gratings were displayed on the 7.76 $\times 6.61 \mathrm{deg}$ faces of two Tektronix 5403/D40 oscilloscopes (P-31 Phosphor) that were optimally aligned such that one face was directly above the other. Gratings were generated in a conventional manner; the luminance of the raster displays was modulated by feeding the output of the various function generators into the $Z$ axes of the oscilloscopes. Schmitt triggers, fed by triangle waves and a variable threshold voltage, were used to generate both the rectangular gratings and the adjustable bar display. The latter was simply a single cycle of a low-frequency $(.13 \mathrm{cpd})$ rectangular grating of approximately $97 \%$ duty cycle for the dark bar and $3 \%$ duty cycle for the light bar. The outputs of the Schmitt triggers were ac coupled to the $Z$ axis inputs so that a constant mean luminance level was maintained regardless of duty cycle or bar width. The 
oscilloscope sweep was triggered by a TTL pulse from the sine wave generator (Tektronix FG501) and by the triangle wave for the rectangular gratings and bar stimuli. Since these triggering triangle waves were also used to generate the latter two stimuli, adjustment of duty cycle or bar width did not change the position of the bars or bar on the display screen.

The mean luminance level and contrast of all gratings and the bar were $5 \mathrm{~cd} / \mathrm{m}^{2}$ and $40 \%$, respectively. Control over exposure durations was accomplished with banks of Lafayette repeat cycle timers (Model 5400A).

\section{Observers}

One of the authors (E.B.) and a paid undergraduate volunteer participated in the experiment. Both observers had normal or corrected-to-normal acuity (Keystone Visual Skills tests).

\section{EXPERIMENT 1: DUTY CYCLE SHIFT}

\section{Procedure}

After $3 \mathrm{~min}$ of light adaptation to a field of uniform luminance $\left(5 \mathrm{~cd} / \mathrm{m}^{2}\right)$, an observer made 10 adjustments of the width of a single bar (presented on the lower oscilloscope) so that it matched the width of one of the bars of a grating on the upper oscilloscope. An observer's fixation was maintained on an LED located in the .5-deg gap between the two CRTs. This 2 cycle/deg (cpd) grating had either a square or sinusoidal luminance profile. When the comparison bar on the lower scope was light, it was vertically aligned with one of the light bars in the grating above it; when the comparison bar was dark, it was aligned with a dark grating bar. Observers were light-adapted again for $3 \mathrm{~min}$ after the 10 adjustments were made.

Adaptation consisted of a 1.5 -min continuous presentation of a $25 \%$ or $75 \%$ duty cycle, 2-cpd rectangular grating, or one of these two rectangular gratings alternating with a field of uniform luminance $\left(5 \mathrm{~cd} / \mathrm{m}^{2}\right)$ every $1.5 \mathrm{sec}$ of a total period of $3 \mathrm{~min}$. (Whether the rectangular grating or field of uniform luminance was presented first in the alternating condition was determined randomly. An adapting grating was always presented on the upper oscilloscope, while on the lower scope, a field of uniform luminance $\left(5 \mathrm{~cd} / \mathrm{m}^{2}\right)$ was displayed. During adaptation, an observer's fixation altered between three LEDs that were placed in the .5-deg gap between the two CRTs.

After the adaptation period, 10 more width matches were made between the single bar on the lower scope and one of the bars contained in the grating presented on the upper scope. Between each match, there was a 20 -sec period of adaptation. With practice, no match took more than $3 \mathrm{sec}$ to make.

\section{Results}

Figure 3 presents the results for Subject E.B. The left panel shows the data for the steady presentation of the adapting rectangular grating, while the right panel shows the data for the presentation of the rectangular grating in alternation with a blank field. In both cases, the adapting grating was visible for a total of $1.5 \mathrm{~min}$.

After adapting to a $25 \%$ duty cycle grating (narrow white, wide black), white bars in the test grating appear wider, while black bars appear narrower. This is true for both steady and alternating conditions and for sine or square wave test gratings. The average shift was $13.5 \%$ (wider) for the white bar and $-12.1 \%$ (narrower) for the black bar. Following adaptation to the $75 \%$ duty cycle grating (narrower black bars, wide white), the shifts were in the opposite direction: black bars were seen as wider by $10.2 \%$ and white
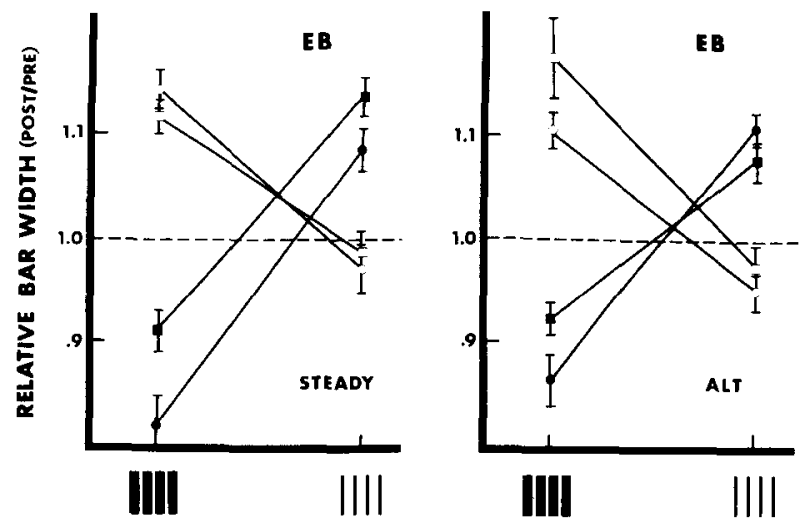

Figure 3. Results of Experiment 1 for Subject E.B. The ordinate represents the postadaptation width setting as a proportion of the preadaptation setting, while the abscissa denotes the duty cycle of the adapting grating. Filled symbols: dark bar adjustments. Open symbols: light bar adjustments. Circles: sine wave test grating. Squares: square wave test grating. Panel on the left shows data for the steady presentation condition; on the right are the data for the alternating condition. Vertical bars indicate one standard error.
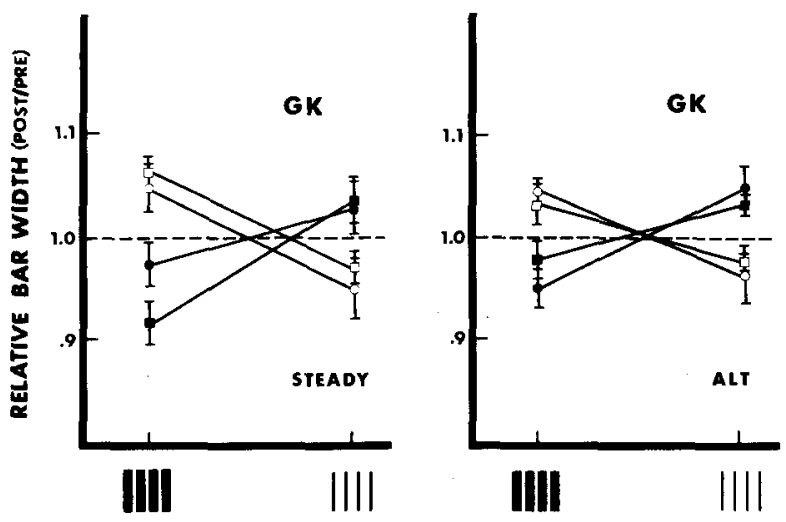

Figure 4. Results of Experiment 1 for Subject G.K. Details same as for Figure 3.

bars as narrower, although here only by $3 \%$. All of these average perceived changes in bar widths are significant at $\mathrm{p}<.01$ (criterion $\mathrm{t}=2.71, \mathrm{df}=39$ ).

The second subject, G.K., showed equivalent results (Figure 4), although the effects are smaller in magnitude. White bars appeared, on the average, $4.6 \%$ wider and black bars appeared $4.0 \%$ narrower following adaptation to the $25 \%$ rectangular grating; white bars appeared 3.8\% narrower and black bars appeared $3.6 \%$ wider following adaptation to the $75 \%$ rectangular grating. All of these average changes in bar width are significant at $p<.01$. Notice that this subject did not show an anomalously small effect for white bars in the $75 \%$ grating condition.

The data indicate that the procedure replicates DeValois' findings when mean luminance is equalized for all stimuli. The magnitude of the effect observed here for Subject G.K. is comparable to that 
reported by DeValois, while the effects for Subject E.B. are substantially larger.

Since the main experiment (Experiment 3) will make use of alternating presentation of rectangular and sine wave gratings and a sine wave test, these conditions here serve as controls for that experiment. Neither the alternating presentation nor the sine wave test affected the results here, and we can assume that the measurements in the final experiment will accurately reflect the duty cycle shift originally studied by DeValois.

\section{EXPERIMENT 2: FREQUENCY SHIFT}

\section{Procedure}

The procedure for this experiment was identical to that of Experiment 1 except that, during the adaptation period, a 2-cpd (1f) or 4-cpd (2f) sine wave grating (on the upper scope) was alternated with a field of uniform luminance $\left(5 \mathrm{~cd} / \mathrm{m}^{2}\right)$ every $1.5 \mathrm{sec}$ for a total time of $3 \mathrm{~min}$.

\section{Results}

Since the widths of white and black bars were judged independently, a frequency shift is indicated when both bar widths change in the same direction. Figure 5 shows the bar-width judgments for adaptation to $1 \mathrm{f}$ and $2 \mathrm{f}$ sine wave gratings alternating with blank fields. For both subjects, the if adaptation produces negligible shifts (average values of $.4 \%$ and $1.4 \%$ for E.B. and G.K., respectively), while the $2 \mathrm{f}$ stimulus produces a substantial shift $(8.0 \%$ and $9.1 \%$, respectively).

The frequency shifts observed here with alternating presentation are quite comparable to those reported elsewhere for steady presentation of the adapting field (e.g., Blakemore \& Sutton, 1969).

\section{EXPERIMENT 3: COMBINED FREQUENCY AND DUTY CYCLE SHIFTS}

\section{Procedure}

The only change in procedure from Experiment 2 was that, dur-
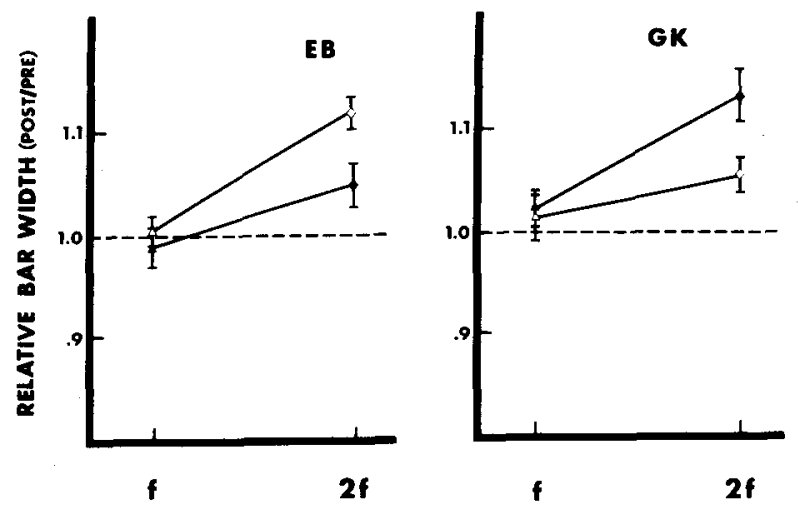

Figure 5. Results of Experiment 2 for Subjects E.B. and G.K. Relative bar width adjustments are shown for adaptation to if or $2 f$ sine wave gratings alternating with a blank field. Filled symbols: dark bar adjustments. Open symbols: light bar adjustments. Triangles: 1f. Diamonds: $2 f$.
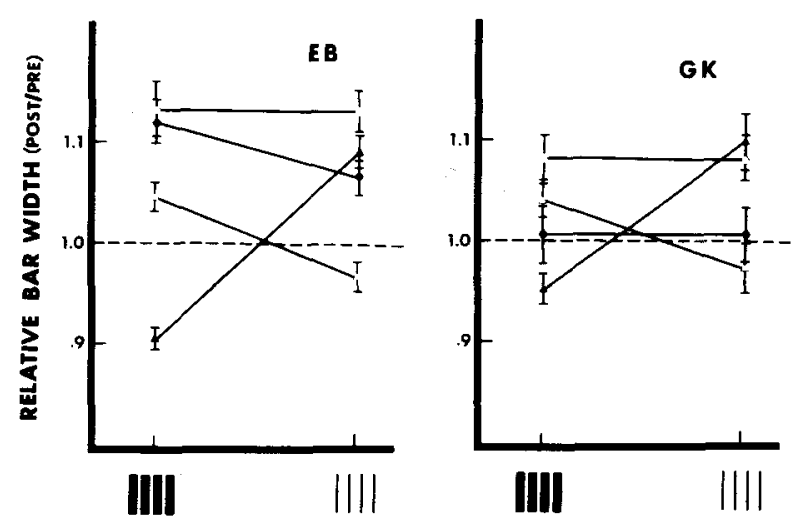

Figure 6. Results of Experiment 3 for E.B. and G.K. Duty cycle of rectangular adapting grating is indicated on the abscissa. The rectangular grating alternated with either a $1 f$ sine wave grating (triangles) or a $2 f$ sine wave grating (diamonds). Filled symbols: dark bar adjustments. Open symbols: light bar adjustments.

ing adaptation, the $1 \mathrm{f}$ or $2 \mathrm{f}$ sine wave gratings were alternated with a $25 \%$ or $75 \%$ duty cycle rectangular grating every $1.5 \mathrm{sec}$ during the $3 \mathrm{~min}$ of initial adaptation and the refresh periods.

\section{Results}

When the rectangular grating is alternated with the If sine wave grating, the pattern of results (Figure 6, triangular symbols) is almost identical to that seen following adaptation to the rectangular grating alone. The procedure of alternating one grating with another during adaptation does not, by itself, seem to interfere with the aftereffects produced by the rectangular grating.

When the rectangular grating is alternated with the $2 \mathrm{f}$ sine wave grating, a completely different pattern of results is seen (Figure 6, diamond symbols). The differential effect of the $25 \%$ and $75 \%$ rectangular gratings on the black and white bar-width judgments disappears. That is, the bar-width judgments for black and white bars are no longer affected by the duty cycle of the adapting rectangular grating. For Subject E.B., some differential effect is seen for the black bar adjustment, but it is in the direction opposite to that predicted by the hypothesis of separate detection of black and white bars. Both E.B. and G.K. show a frequency shift following the combined adaptation. That is, the average shift for both black and white bars is $11.0 \%$ for E.B. and $4.1 \%$ for G.K., although G.K. shows a width shift only for white bar adjustments.

\section{GENERAL DISCUSSION}

Figure 7 shows the data for Experiments 1, 2, and 3 , expressed in terms of frequency shift and duty cycle shift, both averaged over the two subjects. The left-hand panel shows that the effect of the sine wave grating on perceived spatial frequency of the test was not influenced by the presence of the rectangular grating during adaptation. 

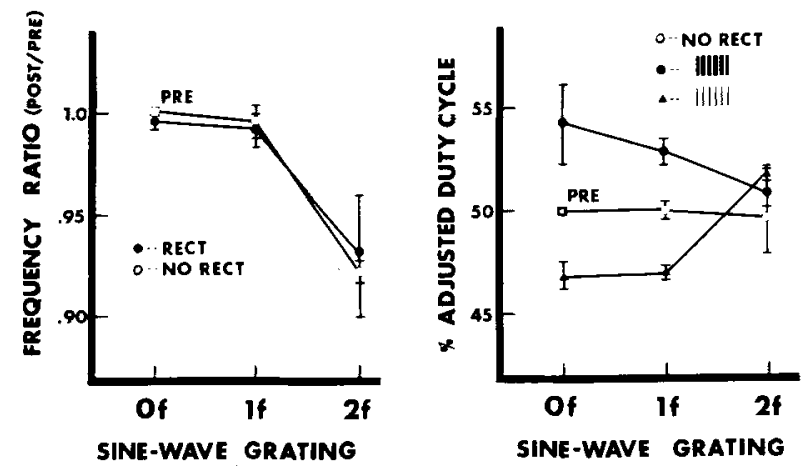

Figure 7. Frequency shift and duty cycle shift resulting from various combinations of rectangular and sine wave adapting gratings presented in alternation. The of sine wave grating indicates a field of uniform luminance. The data are derived from the appropriate conditions of the three experiments reported here and averaged over the two subjects; the vertical bars represent betweensubjects standard errors. The adjusted duty cycle is derived from the pre- and post- dark and light bar adjustments as follows: $D C=$ $(100 \times$ postlight/prelight) $/$ (postlight/prelight) + (postdark/ predark)].

The right-hand panel indicates that the duty cycle shift remains relatively constant, whether generated by adaptation to a rectangular grating alternating with a field of uniform luminance (0f on abscissa; a $7.3 \%$ difference between the effects of the $25 \%$ and $75 \%$ rectangular grating) or alternating with a $1 \mathrm{f}$ grating $(6.0 \%$ difference). When the rectangular grating is alternated with a $2 \mathrm{f}$ sine wave grating, however, the duty cycle shift is eliminated and, in fact, reverses slightly $(-.7 \%)$.

\section{Independent Processing}

The hypothesis of independent analysis of black and white pattern features did not predict this suppression of the duty cycle shift following adaptation to a rectangular grating and a $2 f$ sine wave grating. That is, with a $25 \%$ rectangular grating, the subject sees narrow white bars and wide black bars, while the $2 \mathrm{f}$ grating presents narrow white and black bars. Little or no bar-width shift was expected for black bars, while a significant shift was expected for white bars. Since the reverse was predicted with combined adaptation to a $75 \%$ rectangular grating and a $2 \mathrm{f}$ sine wave (i.e., little or no effect on white bars but a significant effect for black bars), the overall prediction was for the observation of both a frequency shift (nonzero average shift of black and white bars) and a duty cycle shift (differential effect of $25 \%$ and $75 \%$ gratings on black and white bar adjustments).

Although the hypothesis of independent black and white processing certainly appears to be violated in our data, it may be tenable when single bars are stimuli rather than gratings. Adaptation to a single bar might affect primarily orientation-selective simple cells with on-center, off-surround organization for a white bar and off-center on-surround for a black bar.
In this limiting case (approaching $0 \%$ or $100 \%$ duty cycle), then, the interpretation of independent analysis of black and white pattern elements becomes plausible. DeValois (1977) has shown that adaptation to single bars of a given contrast does produce width shifts in test bars of the same contrast but not in test bars of the opposite contrast.

While the independence hypothesis may be reasonable in this special case, our data show that it does not hold in general.

\section{Phase-Sensitive Mechanisms}

Several authors have argued for the presence of wide-band phase-sensitive channels. In particular, Burr (1980) has demonstrated that the threshold for discriminating the relative phase of a first and third harmonic is a fairly constant $30 \mathrm{deg}$ for all spatial frequencies for which both components are clearly visible. When expressed in terms of retinal displacement, however, the threshold varies by a factor of 16 across the same range of frequencies. These data point strongly to mechanisms capable of responding to relative phase as opposed to absolute retinal displacement. Using a masking paradigm, Arend and Lange (1979) have shown that these wide-band phasesensitive mechanisms appear to be organized in parallel with the narrow-band spatial frequency channels rather than taking input from them.

In the context of our experiments, it was proposed that phase-sensitive mechanisms responsive to the first and second harmonics would be adapted selectively by wide white (peaks-subtract phase) or wide black (peaks-add) gratings. The resulting imbalance in responsiveness across the phase-sensitive mechanisms would then lead to a shift in the perceived duty cycle of subsequently viewed square wave gratings. Since a mechanism that responds to combinations of first and second harmonics should also respond to the second harmonic alone, it was argued that prolonged exposure to a $2 \mathrm{f}$ grating would uniformly adapt all these phase-sensitive mechanisms. When these two stimuli are alternated, the uniform reduction due to exposure to $2 \mathrm{f}$ should reduce or eliminate the imbalance due to exposure to the rectangular grating and the duty cycle shift should be suppressed.

Although this prediction was borne out in the data, a companion prediction was not. That is, exposure to the if grating alone should also uniformly reduce the responsiveness of all phase mechanisms responding to combinations of $1 \mathrm{f}$ and $2 \mathrm{f}$. Combining adaptation to $1 \mathrm{f}$ and a rectangular grating should therefore have resulted in suppression of the duty cycle shift. However, exposure to the $1 \mathrm{f}$ grating did not affect the duty cycle shift.

The possibility of phase inhibition suggested by Klein and Stromeyer (1980) may explain this apparent contradiction. They proposed that mechanisms tuned to similar spatial frequencies but having differ- 
ent phase selectivities would be mutually inhibitory. According to Klein and Stromeyer, this phase inhibition would enhance the dominant pattern features at each retinal location. Assuming that the set of channels that is mutually inhibitory should include all those responding to $1 \mathrm{f}$ in combination with the various harmonics, the set should also include channels tuned to $1 \mathrm{f}$ alone, that is, $1 \mathrm{f}$ in combination with the absence of harmonics. ${ }^{1}$

Presentation of a If grating, rather than adapting all channels responding to combinations of $1 \mathrm{f}$ and $2 \mathrm{f}$, would therefore inhibit their activity, masking them from adaptation. Thus, the imbalance in the channels induced by exposure to a rectangular grating would not be diminished by exposure to if, and a duty cycle shift would be observed. The $2 \mathrm{f}$ grating, on the other hand, would activate (not inhibit) channels responding to combinations of $1 \mathrm{f}$ and $2 \mathrm{f}$ and the duty cycle shift would be suppressed here, as previously described.

The combined adaptation used here has demonstrated that black and white pattern features in gratings are not processed independently. Phase-sensitive mechanisms do appear to account for the data when the possibility of inhibition between such channels is considered.

\section{REFERENCES}

Arend, L. E., Jr., \& Lange, R. V. Phase dependent interaction of widely separated spatial frequencies in pattern discrimination. Vision Research, 1979, 19, 1089-1092.

Atrinson, J., \& Campbell, F. W. The effect of phase on the perception of compound gratings. Vision Research, 1974, 14, 159-162.
Blakemore, C., \& Sutton, P. Size adaptation: A new aftereffect. Science, 1969, 166, 245-247.

Burr, D. C. Sensitivity to spatial phase. Vision Research, 1980, 20, 391-396.

Burton, G. J., Nagshineh, S., \& Ruddock, K. H. Processing by the human visual system of the light and dark contrast components of the retinal image. Biological Cybernetics, 1977, 27, 189-197.

DEVALOIS, K. K. Independence of black and white: Phase-specific adaptation. Vision Research, 1977, 17, 209-215.

Klein, S., \& Stromeyer, C. F., III. On inhibition between spatial frequency channels: Adaptation to complex gratings. Vision Research, 1980, 20, 459-466.

Nachmias, J., \& Weber, A. Discrimination of simple and complex gratings. Vision Research, 1975, 15, 217-223.

Sansbury, R. V., Distelhorst, J., \& Moore, S. A phase specific adaptation effect of the square wave grating. Investigative Ophthalmology and Visual Science, 1978, 17, 442-448.

Stromeyer, C. F., III, \& Kle IN, S. Spatial frequency channels in human vision as asymmetric (edge) mechanisms. Vision Research, 1974, 14, 1409-1420.

Stromeyer, C. F., III, Lange, A. F., \& Ganz, L. Spatial frequency phase effect in human vision. Vision Research, 1973, 13, 2345-2360.

\section{NOTE}

1. Klein and Stromeyer (1980) showed that inhibition between symmetric and antisymmetric spatial frequency channels would account for their data on combined adaptation to first and third harmonics. The symmetric and antisymmetric channels (Stromeyer \& Klein, 1974) have similar bandwidths but are sensitive to different phase relationships among the frequencies within their passbands. We are expanding their phase-inhibition suggestion to include inhibition between channels of varying bandwidth: narrowband channels tuned to $1 f$ and wide-band channels tuned to $1 f$ in combination with various harmonics. The rationale of emphasizing dominant local features remains the same and is actually enhanced by the increased generality proposed here.

(Received for publication August 20, 1980; revision accepted March 12, 1981.) 